\title{
Blocking Wythoff Nim
}

\author{
Urban Larsson \\ Mathematical Sciences \\ Chalmers University of Technology and University of Gothenburg \\ Göteborg, Sweden \\ urban.larsson@chalmers.se
}

Submitted: Oct 27, 2010; Accepted: May 5, 2011; Published: May 23, 2011

Mathematics Subject Classification: 91A46

\begin{abstract}
The 2-player impartial game of Wythoff Nim is played on two piles of tokens. A move consists in removing any number of tokens from precisely one of the piles or the same number of tokens from both piles. The winner is the player who removes the last token. We study this game with a blocking maneuver, that is, for each move, before the next player moves the previous player may declare at most a predetermined number, $k-1 \geq 0$, of the options as forbidden. When the next player has moved, any blocking maneuver is forgotten and does not have any further impact on the game. We resolve the winning strategy of this game for $k=2$ and $k=3$ and, supported by computer simulations, state conjectures of 'sets of aggregation points' for the $P$-positions whenever $4 \leq k \leq 20$. Certain comply variations of impartial games are also discussed.
\end{abstract}

\section{Introduction}

We study variations of the 2-player combinatorial game of Wythoff Nim [Wyt07]. This game is impartial, since the set of options of a given position does not depend on which player is in turn to move. A background on such games can be found in [ANW07, BCG82, Con76]. Let $\mathbb{N}$ and $\mathbb{N}_{0}$ denote the positive and non-negative integers respectively and let the 'game board' be $\mathcal{B}:=\mathbb{N}_{0} \times \mathbb{N}_{0}$.

Definition 1. Let $(x, y) \in \mathcal{B}$. Then $(x-i, y-j)$ is an option of Wythoff Nim if either:

(v) $0=i<j \leq y$,

(h) $0=j<i \leq x$,

(d) $0<i=j \leq \min \{x, y\}$,

$i, j \in \mathbb{N}_{0}$. 
In this definition one might want to think about $(\mathrm{v}),(\mathrm{h})$ and $(\mathrm{d})$ as symbolizing the 'vertical' $(0, i)$, 'horizontal' $(i, 0)$ and 'diagonal' $(i, i)$ moves respectively. Two players take turns in moving according to these rules. The player who moves last (that is to the position $(0,0))$ is declared the winner. Here we study a variation of Wythoff Nim with a blocking maneuver [SmSt02, HoRe1].

Notation 1. The player in turn to move is called the next player and the other player the previous player.

Definition 2. Let $k \in \mathbb{N}$ and let $G$ denote an impartial game. In the game of $G^{k}$, the blocking- $k$ variation of $G$, the options are the same as those of $G$. But before the next player moves, the previous player may declare at most $k-1$ of them as forbidden. When the next player has moved, any blocking maneuver is forgotten and has no further impact on the game. The player who moves last (to a non-blocked position) is declared the winner. We call the game $W^{k}$, Blocking-k Wythoff Nim.

Clearly, by this definition, since $G$ is impartial, $G^{k}$ is also. Further, if $G$ does not have any draw positions neither does $G^{k}$. (On the other hand a draw-free $G^{k}$ does not imply the same for $G$.) Hence $W^{k}$ does not contain any draw positions and so, as usual, we partition the positions into $P$ and $N$, the previous and next player winning positions respectively.

Definition 3. Let $G$ be an impartial game without draw positions. Then the value of (a position of) $G^{k}$ is $P$ if strictly less than $k$ of its options are $P$, otherwise it is $N$. Denote by $\mathcal{P}_{k}$ the set of $P$-positions of $\mathrm{W}^{k}$.

By this definition, the next player wins if and only if the position is $N$. It leads to a recursive definition of the set of $P$-positions of $\mathrm{W}^{k}$, see also Proposition 1.2 on page 4 . Since both the Wythoff Nim type moves and the blocking maneuvers are 'symmetric' on the game board, it follows that the sets of $P$ - an $N$-positions are also 'symmetric'. Hence we have the following notation.

Notation 2. The 'symmetric' notation $\{x, y\}$ for unordered pairs of non-negative integers is used whenever the positions $(x, y)$ and $(y, x)$ are equivalent. Two positions are equivalent if and only if they have the same Grundy values.

Let us explain the main results of this paper, see also Figure 2.

Definition 4. Let $\phi=\frac{1+\sqrt{5}}{2}$ denote the Golden ratio. Then

$$
\begin{aligned}
& \mathcal{R}_{1}:=\left\{\left\{\lfloor\phi n\rfloor,\left\lfloor\phi^{2} n\right\rfloor\right\} \mid n \in \mathbb{N}_{0}\right\}, \\
& \mathcal{R}_{2}:=\{(0,0)\} \cup\left\{\{n, 2 n+1\} \mid n \in \mathbb{N}_{0}\right\} \cup\left\{(2 x+2,2 y+2) \mid(x, y) \in \mathcal{R}_{1}\right\}, \text { and } \\
& \left.\mathcal{R}_{3}:=\{(0,0)\} \cup\left\{\{n, 2 n+1\},\{n, 2 n+2\} \mid n \in \mathbb{N}_{0}\right\}\right\} .
\end{aligned}
$$

Theorem 1.1. Let $i \in\{1,2,3\}$. Then $\mathcal{P}_{i}=\mathcal{R}_{i}$. 

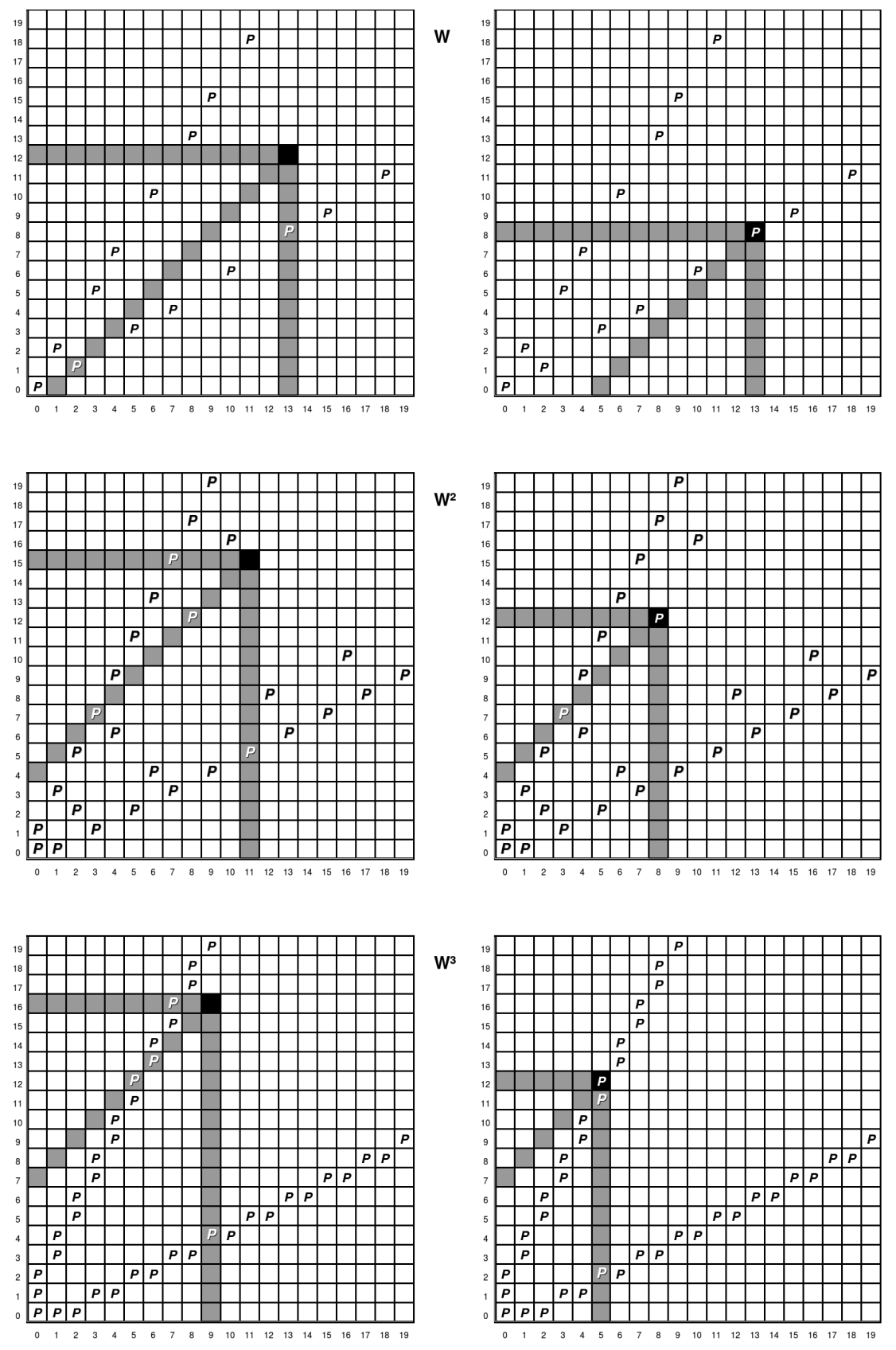

Figure 1: The two figures at the top illustrate options of two instances of Wythoff Nim together with its initial $P$-positions. The middle and lower couples of figures represent $\mathrm{W}^{2}$ and $\mathrm{W}^{3}$ respectively. For example in the middle left figure the 'gray' shaded positions are the options of the 'black' $N$-position $(11,15)$. This position is $N$ since, by rule of game, only one of the two $P$-positions in its set of options can be forbidden. In contrast, the position $(8,12)$ is $P$ (middle-right) since there is precisely one single $P$-position in its set of options. It can (and will) be forbidden. 
It is well known that the set $\mathcal{P}_{1}=\mathcal{R}_{1}$ [Wyt07]. We prove the latter two results in Section 2. In Section 3 we discuss a certain family of 'comply games'. In particular we define the game $\mathrm{W}_{k}$ and prove that its set of $N$-positions is identical to $\mathcal{P}_{k}, k \in \mathbb{N}$. In Section 4 we discuss some experimental results and provide a table of conjectured sets of aggregation points of $\mathcal{P}_{k}$ for each $k \in\{4,5, \ldots, 20\}$.

\subsection{Some general results}

The set $\mathcal{R}_{1}$ has some frequently studied properties. Namely, the sequences $(\lfloor\phi n\rfloor)$ and $\left(\left\lfloor\phi^{2} n\right\rfloor\right)$ are so-called complementary sequences of $\mathbb{N}$, e.g. [Fra82], that is they partition $\mathbb{N}$. (This follows from the well known 'Beatty's theorem' [Bea26].) In this paper we make use of a generalization of this concept - often used in the study of so called '(exact) covers by Beatty sequences' e.g. [Fra73, Gra73, Heg1].

Definition 5. Let $p \in \mathbb{N}$. Suppose that $A$ is a set of a finite number of sequences of non-negative integers. Then $A$ is a $p$-cover (cover if $p=1$ ) of another set, say $S \subset \mathbb{N}_{0}$, if, for each $x \in S$, the total number, $\xi(A, S, x)$, of occurrences of $x$, in the sequences of $A$, exceeds or equals $p$. Further, $A$ is an exact $p$-cover of $S$ if, for all $x, \xi(A, S, x)=p$.

The special case of $S=\mathbb{N}, \# A=2$ and $p=1$ in this definition is 'complementarity'. For general $p$ and with $\# A=2$ the term $p$-complementarity is used in [Lar1].

Let us begin by giving some basic results valid for general $\mathrm{W}^{k}$.

Proposition 1.2. Let $k \in \mathbb{N}$ and define $\left\{\left\{a_{i}, b_{i}\right\} \mid i \in \mathbb{N}_{0}\right\}=\mathcal{P}_{k}$, where, for all $i, a_{i} \leq b_{i}$ and the ordered pairs $\left(a_{i}, b_{i}\right)$ are in lexicographic order, that is $\left(a_{i}\right)$ is non-decreasing and $a_{i}=a_{j}$ together with $i<j$ imply $b_{i}<b_{j}$. Then,

(i) $\left(a_{x}, b_{x}\right)=(0, x)$ if and only if $x \in\{0,1, \ldots, k-1\}$,

(ii) the set $\left\{\left(a_{i}\right),\left(b_{i}\right) \mid i \geq k\right\}$ is an exact $k$-cover of $\mathbb{N}$,

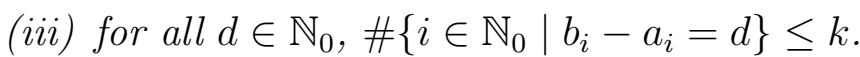

Proof. The case $k=1$ follows from well known results on Wythoff Nim [Wyt07]. Hence, let $k>1$. The item (i) is obvious (see also (2)). For (ii) suppose that there is a least $x^{\prime} \in \mathbb{N}$ such that

$$
r=\#\left(\left\{i \mid a_{i}=x^{\prime}\right\} \cup\left\{i \mid b_{i}=x^{\prime}\right\}\right) \neq k .
$$

Clearly, by the blocking rule, this forces $r<k$ for otherwise there trivially exists a nonblocked Nim-type move $\boldsymbol{x} \rightarrow \boldsymbol{y}$, where both $\boldsymbol{x}, \boldsymbol{y} \in \mathcal{P}_{k}$. Suppose that $y$ is the largest integer such that $\left(x^{\prime}, y\right) \in \mathcal{P}_{k}$. Then, by the blocking rule, for all integers

$$
z>y
$$

there must exist a $P$-position in the set of horizontal and diagonal options of $\left(x^{\prime}, z\right)$. (For otherwise all $P$-positions in the set of options of $\left(x^{\prime}, z\right)$ could be blocked off.) But, by assumption, the total number of $P$-positions in the columns $0,1, \ldots, x^{\prime}-1$ is precisely 
$k\left(x^{\prime}-1\right)$ and each such position is an option of precisely two positions in column $x^{\prime}$, which contradicts (1). Item (iii) is obvious by Definition 2.

Notation 3. A position (of $\mathrm{W}^{k}$ ) is terminal if all options may be blocked off by the previous player.

A player who moves to a terminal position may, by Definition 2, be declared the winner. Let $k \in \mathbb{N}$. The terminal positions of $\mathrm{W}^{k}$ are given by the following result. We omit the elementary proof.

Proposition 1.3. Let $k \in \mathbb{N}$. The set of terminal positions of $\mathrm{W}^{k}$ is precisely

$$
\mathcal{T}(k):=\left\{\{x, y\} \mid x \leq y<k-2 x, x, y \in \mathbb{N}_{0}\right\} .
$$

The set $\mathcal{T}(k)$ is a lower ideal, that is $(x, y) \in \mathcal{T}(k)$ implies $(x-i, y-j) \in \mathcal{T}(k)$, for all $i \in\{0,1, \ldots, x\}$ and all $j \in\{0,1, \ldots, y\}$. The number of positions in this set is

$$
\# \mathcal{T}(k):= \begin{cases}3(m+1)^{2}-2(m+1) & \text { if } k=3 m+1 \\ 3(m+1)^{2} & \text { if } k=3 m+2 \\ 3(m+1)^{2}+2(m+1) & \text { if } k=3(m+1)\end{cases}
$$

$m \in \mathbb{N}_{0}$.

In particular, the set of terminal positions of $\mathrm{W}^{2}$ and $\mathrm{W}^{3}$ are $\mathcal{T}(2)=\{(0,0),\{0,1\}\}$ $(\# \mathcal{T}(2)=3)$ and $\mathcal{T}^{3}=\{(0,0),\{0,1\},\{0,2\}\}(\# \mathcal{T}(3)=5)$ respectively.

Before we give the proof of the main results, let us provide some background on blocking maneuvers on 'Nim-type' games.

\subsection{Some background}

In [HoRe01] a blocking maneuver of the classical game of Nim [Bou02] is proposed: The game of "Blocking Nim" proceeds in exactly the same way as ordinary Nim, except that for each pile of counters the previous player has the option to specify a number of counters which may not be removed. A very close connection to the winning strategy of regular Nim is demonstrated. (Note that in this way several moves may be blocked off at each stage of the game.)

In [HoRe] the authors study 3-pile Nim with a blocking maneuver of the type, "exactly one move can be blocked off at each stage of the game" and demonstrates that "the winning strategy for the more complicated version is much simpler than for ordinary Nim". However, the authors explain that they do not know how to extend the result to games with more then three piles or to games with more than one blocking maneuver. Since we could not find the solution of the corresponding game on two piles in the literature, we include it here. We omit the inductive proof, which is by analogy with that of the main result of this paper in Section 2 (but here we obviously do not consider (d) type moves). 
Proposition 1.4. Let the game be a variation of Nim on two piles of counters where at most $k-1$ moves, $k \in \mathbb{N}$, may be blocked off at each stage. Then the P-positions are of the form $\{x, y\}$, where either $|y-x|<k$ and $y-x \equiv k-1(\bmod 2)$ or $x+y<k$. The case $k=1$ corresponds to regular Nim on two piles.

In [SmSt02, GaSt04], the authors study several comply/constrain variations of the classical game of Nim on several piles of the type, the previous player puts a constraint of removing $x(\bmod n)$ tokens, for a given $2 \leq n \in \mathbb{N}$. They show that the $P$-positions of such games are 'close' to those of regular Nim.

Various blocking maneuvers on Wythoff Nim have been studied in [FrPe, Gur10, HeLa06, Lar1, Lar09]. We will return to some of these games in Section 3. Connections of the set of $P$-position to exact $p$-covers of Beatty-type non-decreasing sequences of integers are demonstrated. (Hence the $P$-positions of these games are 'close' to those of Wythoff Nim in some sense.)

\section{Proof of the main result}

Given a blocking parameter $k=2$ or 3 and a position $(x, y)$, we count the total number of options contained in our candidate set of $P$-positions $\mathcal{R}_{2}$ or $\mathcal{R}_{3}$ respectively. Then we derive the value of $(x, y)$ as follows. The previous player will win if and only if the total number of options in the candidate set is strictly less than $k$. Hence, let us define some functions, counting the number of options in some specific 'candidate set' and of the specific types, (v), (d) and (h) respectively.

Definition 6. Let $(x, y) \in \mathcal{B}$. Given a set $S \subset \mathcal{B}$, let us define

$$
\begin{aligned}
v_{x, y} & =v_{x, y}(S):=\#(\{(w, y) \mid x>w \geq 0\} \cap S), \\
d_{x, y} & =d_{x, y}(S):=\#(\{(w, z) \mid x-w=y-z>0\} \cap S), \\
h_{x, y} & =h_{x, y}(S):=\#(\{(x, z) \mid y>z \geq 0\} \cap S), \\
f_{x, y} & =f_{x, y}(S):=d_{x, y}+v_{x, y}+h_{x, y},
\end{aligned}
$$

$w, z \in \mathbb{N}_{0}$.

Notation 4. We use the notation $\left(x_{1}, x_{2}\right) \rightarrow\left(y_{1}, y_{2}\right)$ if there is a Wythoff Nim (Definition 1) type move from $\left(x_{1}, x_{2}\right)$ to $\left(y_{1}, y_{2}\right)$.

\subsection{Proof of Theorem 1.1.}

With notation as in Definition 4 and 6 , put $S=\mathcal{R}_{2}$ and let $k=2$. Hence, we consider the game $\mathrm{W}^{2}$. Then, by the blocking rules in Definition 2, each $P$-position has the property that at most one of its options is $P$ and each $N$-position has the property that at least two $P$-position are in its set of options. Thus, the theorem holds if we can prove that the value of $(x, y) \in \mathcal{B}$ is $P$ if and only if $f_{x, y}\left(\mathcal{R}_{2}\right) \leq 1$. Hence notice that (see also (2)) 
- $f_{0,0}<f_{0,1}=1$ and $(0,0),\{0,1\}$ are $P$,

- $x \geq 2, f_{0, x} \geq 2$ and $\{0, x\}$ is $N$,

- $f_{1,1}=3$ and $(1,1)$ is $N$,

- $f_{1,2}=2$ and $\{1,2\}$ is $N$.

Further, the 'least' $P$-position which is not terminal is $(2,2)$, namely $f_{2,2}=1$ since, by the above items, the only option which is a $P$-position is $(0,0)$.

We divide the rest of the proof of the strategy of $\mathrm{W}^{2}$ into two 'classes' depending on whether $(x, y) \in \mathcal{B}$ belongs to $\mathcal{R}_{2}$ or not.

Suppose that $(x, y) \in \mathcal{R}_{2}$. That is, we have to prove that $f_{x, y}\left(\mathcal{R}_{2}\right) \leq 1$. We are done with the cases $(x, y)=(0,0),(0,1)$ and $(2,2)$. We may assume that $1 \leq x \leq y$.

Case 1: Suppose that $y=2 x+1$. Then, we claim that $h_{x, y}=0, d_{x, y}=0$ and $v_{x, y}=1$.

Proof. The horizontal options of $(x, 2 x+1)$ are of the form $(z, 2 x+1)$ with $z<x$. But all positions $(r, s)$ in $\mathcal{R}_{2}$ satisfy

$$
s \leq 2 r+1 .
$$

This gives $h_{x, y}=0$.

The diagonal options are of the form $(z, x+z+1)$, with $0 \leq z<x$. Again, by (3), this gives $d_{x, y}=0$.

For the vertical options, if $x \leq 2$, we are done, hence suppose $x>2$. Then, we may use that $\left\{(2\lfloor\phi n\rfloor+2)_{n \in \mathbb{N}},\left(2\left\lfloor\phi^{2} n\right\rfloor+2\right)_{n \in \mathbb{N}},(2 n+1)_{n \in \mathbb{N}}\right\}$ is an exact cover of $\{3,4,5, \ldots\}$.

Namely, if $x:=2 z+1$ is odd, we have that

$$
y=2 x+1>x=2 z+1>z,
$$

so that $(x, y) \rightarrow(2 z+1, z) \in \mathcal{R}_{2}$. Since $x$ is odd, any vertical option in $\mathcal{R}_{2}$ has to be of this form.

If, on the other hand, $x:=2 z \geq 2$ is even, then, since (by [Wyt07]) $(\lfloor\phi n\rfloor)_{n \in \mathbb{N}}$ and $\left(\left\lfloor\phi^{2} n\right\rfloor\right)_{n \in \mathbb{N}}$ are complementary, there is precisely one $n$ such that either $z=\lfloor\phi n\rfloor+1$ or $z=\left\lfloor\phi^{2} n\right\rfloor+1$. For the first case

$$
\begin{aligned}
(x, 2 x+1) & =(2 z, 4 z+1) \\
& =\left(2\lfloor\phi n\rfloor+2,4\left\lfloor\phi^{2} n\right\rfloor+3\right) \rightarrow\left(2\lfloor\phi n\rfloor+2,2\left\lfloor\phi^{2} n\right\rfloor+2\right) \in \mathcal{R}_{2} .
\end{aligned}
$$

The second case is similar. But, since $x$ is even, any option in $\mathcal{R}_{2}$ has to be precisely of one of these forms. We may conclude that $v_{x, y}=1$.

Case 2: Suppose that $x=2\lfloor\phi n\rfloor+2$ and $y=2\left\lfloor\phi^{2} n\right\rfloor+2$, for some $n \in \mathbb{N}_{0}$. Then, we claim that $d_{x, y}=1$ and $v_{x, y}=h_{x, y}=0$. 
Proof. If $n=0$, we are done, hence suppose that $n>0$. We have that

$$
(2\lfloor\phi n\rfloor+2,2\lfloor\phi n+n\rfloor+2)-(2 n-1,4 n-1)=(2\lfloor\phi n\rfloor-2 n+3,2\lfloor\phi n\rfloor-2 n+3)
$$

is a diagonal move in Wythoff Nim (and where the '-'sign denotes vector subtraction). This gives $d_{x, y} \geq 1$. We may partition the differences of the coordinates of the positions in $\mathcal{R}_{2}$ into two sequences,

$$
((2 n+1)-n)_{n \in \mathbb{N}_{0}}=(n)_{n \in \mathbb{N}}
$$

and

$$
\left(2\left\lfloor\phi^{2} n\right\rfloor+2-(2\lfloor\phi n\rfloor+2)\right)_{n \in \mathbb{N}_{0}}=(2 n)_{n \in \mathbb{N}}
$$

respectively. These sequences are strictly increasing, which gives $d_{x, y}=1$.

For the second part we may apply the same argument as in Case 1, but in the other direction. Namely, $2 x+1 \geq 4\lfloor\phi n\rfloor+3>2\left\lfloor\phi^{2} n\right\rfloor+2>2\lfloor\phi n\rfloor+2$, which implies that all Nim-type options belong to the set $\mathcal{B} \backslash \mathcal{R}_{2}$.

We are done with the first class. Hence assume that $(x, y) \notin \mathcal{R}_{2}$. That is, we have to prove that $f_{x, y}\left(\mathcal{R}_{2}\right) \geq 2$.

Case 3: Suppose $y>2 x+1$. Then we claim that $v_{x, y}=2, h_{x, y}=0$ and $d_{x, y}=0$.

Proof. By the first argument in Case 1, the latter two claims are obvious. Notice that the set of sequences $\left\{(n)_{n \in \mathbb{N}},(2 n+1)_{n \in \mathbb{N}_{0}},(2\lfloor\phi n\rfloor+2)_{n \in \mathbb{N}_{0}},\left(2\left\lfloor\phi^{2} n\right\rfloor+2\right)_{n \in \mathbb{N}_{0}}\right\}$ constitute an exact 2 -cover of $\mathbb{N}$. This gives $v_{x, y}=2$.

Case 4: Suppose $0<x \leq y<2 x+1$. Then, we claim that either

(i) $d_{x, y}=1$ and $h_{x, y}+v_{x, y} \geq 1$, or

(ii) $d_{x, y}=2$.

Proof. We consider three cases.

(a) $y>\phi x$

(b) $y<\phi x$ and $y-x$ even,

(c) $y<\phi x$ and $y-x$ odd.

In case (a), $v_{x, y}=1$ is verified as in Case 1 . For $d_{x, y}=1$, it suffices to demonstrate that $(x, y)-(z, 2 z+1)=(x-z, y-2 z-1)$, is a legal diagonal move for some $z \in \mathbb{N}_{0}$. Thus, it suffices to prove that $x-z=y-2 z-1$ holds together with $0<z<x$ and $2 z+1<y$. But this follows since the definition of $y$ implies $z+1=y-x \leq 2 x-x=x$.

In case (b) we get $d_{x, y}=2$ by $\left(2\left\lfloor\phi^{2} n\right\rfloor-2\lfloor\phi n\rfloor\right)_{n \in \mathbb{N}}=(2 n)$ and an analog reasoning as in the latter part of (a). (Hence this is (ii).)

In case (c) we may again use the latter argument in (a), but, for parity reasons, there are no diagonal options of the first type in (b), hence we need to return to case (i) and 
thus verify that $h_{x, y}+v_{x, y} \geq 1$. Since $y-x$ is odd we get that precisely one of $x$ or $y$ must be of the form $2 z+1, z \in \mathbb{N}_{0}$. Suppose that $z<x=2 z+1<y$. Then $(x, y) \rightarrow(2 z+1, z)$ gives $v_{x, y} \geq 1$. If, on the other hand, $x \leq y=2 z+1<\phi x$, then $(x, y) \rightarrow(z, 2 z+1)$ is legal since $z<\frac{\phi x-1}{2}<x$, which gives $h_{x, y} \geq 1$.

We are done with $\mathrm{W}^{2}$ 's part of the proof. Therefore, let $S=\mathcal{R}_{3}, k=3$ and consider the game $\mathrm{W}^{3}$. Then one needs to prove that $(x, y) \in \mathcal{R}_{3}, x \leq y$, if and only if $f_{x, y}\left(\mathcal{R}_{3}\right) \leq 2$. Suppose that $(x, y) \in \mathcal{R}_{3}$ with $x \leq y$. Then we claim that $d_{x, y} \leq 1, h_{x, y}=0$ and $v_{x, y} \leq d_{x, y}+1$. Otherwise, if $(x, y) \notin \mathcal{R}_{3}$ and $y>2 x+2$, then we claim that $v_{x, y}=3$, or, if $y<2 x+1$, then we claim that $h_{x, y}=v_{x, y}=1$ and $d_{x, y} \geq 1$. Each case is almost immediate by definition of $\mathcal{R}_{3}$ and Figure 2 , hence we omit further details.

\section{Comply- versus blocking-games}

Let us define a 'comply' variation of any impartial game, which constitutes a subtle variation to that in [SmSt02].

Definition 7. Let $G$ be an impartial game and let $k \in \mathbb{N}$. Then $G_{k}$ denotes the following comply variation of $G$. The previous player is requested to propose at least $k$ of the options of $G$ as allowed next-player moves in $G_{k}$ (and these are all moves). After the next player has moved, this 'comply-maneuver' is forgotten and has no further impact on the game. The last player to propose at least $k$ next-player options is declared the winner.

Clearly this definition gives a recursive definition of all $P$ and $N$ positions of $G_{k}$ (and there are no draw positions). At each stage of the game, the next player wants to find a $P$-position among $k$ proposed options, to move to. Hence, we get the following definition.

Definition 8. Let $G$ be an impartial game. Then the value of (a position of) $G_{k}$ is $N$ if strictly less than $k$ of its options are $N$, otherwise it is $P$.

Thus, as an example, let us regard the comply-variation of one-pile Nim where the previous player has to propose at least one option. In this game the empty pile is $N$ (the previous player loses because he cannot propose any option). Each non-empty pile is $P$, since the previous player will propose the empty pile as the only available option for the next player. Recall that the only P-position of Nim (without blocking maneuver) is the empty pile.

Motivated by this simple example, let us establish that, for all impartial games $G$ (without draw positions), the game $G_{k}$ in Definition 7 has the 'reverse' winning strategy as that of $G^{k}$ in Definition 2. The proof is 'abstract nonsense', immediate by Definitions 3 and 8 .

Proposition 3.1. Let $G$ denote an impartial game. Then the set of P-positions of $G_{k}$ constitute precisely the set of $N$-positions of $G^{k}$.

Proof. Suppose that $x$ is $P$ in $G^{k}$. For this case, we have to demonstrate that $x$ is $N$ in $G_{k}$. By Definition 3, we have that there are at most $k-1$ options of $x$ which are $P$ 
in $G^{k}$. The crucial point is that, by the recursive definition of $P$ and $N$ positions in the respective games and since the options are the same, we get that there are at most $k-1$ options of $x$ which are $N$ in $G_{k}$. By Definition 8 , this gives that $x$ is $N$ in $G_{k}$.

Suppose, on the other hand, that $x$ is $N$ in $G^{k}$. Then we have to demonstrate that $x$ is $P$ in $G_{k}$, that is, that the previous player can propose at least $k$ positions which are $N$ in $G_{k}$. By the definition of an $N$-position in $G^{k}, x$ has at least $k$ options which are $P$ in $G^{k}$. By the recursive definitions of $P$ and $N$ in the respective game and since the options are the same, this corresponds to at least $k$ options which are $N$ in $G_{k}$.

This discussion motivates why we, in the definition of $\mathrm{W}^{k}$ (Definition 2), let the previous player forbid $k-1$, rather than $k$ options. To propose at least $k$ options is the 'complement' of forbidding strictly less than $k$ options - and it is not a big surprise that the set of $P$-positions of $\mathrm{W}^{k}$ are 'complementary' to those of $\mathrm{W}_{k}$. (Another more 'algorithmic' way of thinking of this choice of notation is that (the position of) $\mathrm{W}^{k}$ a priory belongs to the set of forbidden options.) Let us recall some other blocking variations of Wythoff Nim.

Definition 9. Let $k \in \mathbb{N}$. In the game of $k$-blocking Wythoff Nim [HeLa06, Lar09, FrPe], the blocking maneuver constrains at most $k-1$ moves of type (d) in Definition 1 . Otherwise the rules are as in Wythoff Nim. We denote this game by $\mathrm{W}^{k} N$. In another variation, the game of Wythoff $k$-blocking Nim [Lar1], the blocking maneuver constrains at most $k-1$ Nim-type moves that is, of type ( $h$ ) or (v). Denote this game by $\mathrm{WN}^{k}$.

Both these game families are actually defined, and solved, as restrictions of $m$-Wythoff Nim, [Fra82].

Motivated by Proposition 3.1 and the results for $\mathrm{W}^{k}$ and $\mathrm{W}_{k}$, let us round off this section by defining the corresponding 'comply rules' of $\mathrm{W}^{k} \mathrm{~N}$ and $\mathrm{WN}^{k}$. That is, we look for rules of games, say $\mathrm{WN}_{k}$ and $\mathrm{W}_{k} \mathrm{~N}$, such that the $P$-positions of these games correspond precisely to the $N$-positions of $\mathrm{WN}^{k}$ and $\mathrm{W}^{k} \mathrm{~N}$ respectively.

Definition 10. Let the options of $\mathrm{W}^{k} N$ and $\mathrm{W} N^{k}$ be as in Wythoff Nim.

The comply rule for $\mathrm{W}_{k} N$ is: The previous player must propose at least $k$ next player options of type (d) or at least one Nim-type option, that is of type ( $h$ ) or (v).

The comply rule for $\mathrm{WN}_{k}$ is: The previous player must propose at least $k$ Nim-type options or at least one option of type (d).

A player who fails to obey the comply-rule loses.

In the proof of the next proposition, we let the obvious generalizations of Definition 3 and 8 remain implicit. Also we omit the proof for $\mathrm{WN}_{k}$, since it is similar to that of $\mathrm{W}_{k} \mathrm{~N}$.

Proposition 3.2. The P-positions of $\mathrm{WN}^{k}$ correspond precisely to the $N$-positions of $\mathrm{W} N_{k}$ and the P-positions of $\mathrm{W}^{k} N$ correspond precisely to the $N$-positions of $\mathrm{W}_{k} N$.

Proof. A terminal $P$-position of $\mathrm{W}^{k} \mathrm{~N}$ has at most $k-1$ type (d) options and no Nim-type option. This gives that $(0,0)$ is the only terminal position. Clearly $(0,0)$ is $N$ in $\mathrm{W}_{k} \mathrm{~N}$ since the previous player is not able to obey the comply rules. 
Suppose that $(x, y)$ is non-terminal $P$ in $\mathrm{W}^{k} \mathrm{~N}$. Then there are at most $k-1(\mathrm{~d})$ type $P$-positions and no Nim-type $P$-position at all in the set of options of $(x, y)$. We have to demonstrate that $(x, y)$ is $N$ in $\mathrm{W}_{k} \mathrm{~N}$, that is that the previous player cannot propose $k$ (d)-type positions, all of them $N$, neither can he propose a single Nim-type $N$-position. As in the proof of Proposition 3.1, we may assume that an option of $(x, y)$ is $P$ in $\mathrm{W}^{k} \mathrm{~N}$ if and only if it is $N$ in $\mathrm{W}_{k} \mathrm{~N}$. That is, for the game $\mathrm{W}_{k} \mathrm{~N}$, we get that there are at most $k-1$ (d) type $N$-positions and no Nim-type $N$-position at all in the set of options of $(x, y)$. Then, by obeying the comply rules, the previous player has to propose at least one $P$-position for the next player to move to, which gives that $(x, y)$ is $N$ in $\mathrm{W}_{k} \mathrm{~N}$.

Suppose, on the other hand that $(x, y)$ is non-terminal $N$ in $\mathrm{W}^{k} \mathrm{~N}$. Then we have to show that $(x, y)$ is $P$ in $\mathrm{W}_{k} \mathrm{~N}$. By the definition of $N$ in $\mathrm{W}^{k} \mathrm{~N}$, there is at least one Nim-type $P$-position in the set of options of $(x, y)$, or at least $k P$-positions in its set of type $(\mathrm{d})$ options. In the game $\mathrm{W}_{k} \mathrm{~N}$, this means that there is at least one Nim-type $N$ position in the set of options of $(x, y)$ or at least $k N$-positions in its set of type (d) options. Then the previous player is able to obey the comply rules and at the same time force the next player to move to an $N$-position. This gives that $(x, y)$ is $P$ in $\mathrm{W}_{k} \mathrm{~N}$.

One might want to extend the ideas in this proposition to the generalized setting of impartial blocking games in [HoRe1].

\section{Discussion}

One obvious direction of future research is to try and describe the $P$-positions of the games $\mathrm{W}^{k}, k \geq 4$. Let $k \in \mathbb{N}$ and, as in Proposition 1.2 , let $\left\{\left\{a_{i}, b_{i}\right\} \mid i \in \mathbb{N}_{0}\right\}=\mathcal{P}_{k}$ denote the set of $P$-positions of $\mathrm{W}^{k}$. Since this task appears quite hard it seems reasonable to start off by looking for a simpler (but non-exhaustive) classification. For example one would like to know whether the limit

$$
\lim _{i \rightarrow \infty} \frac{b_{i}}{a_{i}}
$$

exists or not, for a given $k$. If we were not able to answer this question, then the tractability (in the sense of [Fra04]) of a game's winning strategy would seem very remote indeed. If the limit does exist, a natural question is if the sequences $\left(a_{i}\right)$ and $\left(b_{i}\right)$ are approximately linear (in the sense of [FrPe]). In this case there might be tools available from (or related to) $[\mathrm{FrPe}, \mathrm{LaWä}]$ to settle a polynomial time winning strategy.

If the limit in (4) does not exist, then we wonder if the set of aggregation points of $\lim _{i \rightarrow \infty} \frac{b_{i}}{a_{i}}$ is finite. More precisely: For some (least) $2 \leq l=l(k) \in \mathbb{N}$, does there exist $l$ sequences $t^{j}, j \in\{1,2, \ldots, l\}$, such that pairwise distinct asymptotic limits

$$
\lim _{i \rightarrow \infty} \frac{b_{t_{i}^{j}}}{a_{t_{i}^{j}}}
$$

THE EleCtronic journal of Combinatorics 18 (2011), \#P120 
exist? We conjecture that, for each $k \in\{4,5, \ldots, 20\}$ in Table $1, l(k)$ is given by the number of entries in row $k$. (Again, if these conjectures hold, it would be interesting to try and obtain more information on the winning strategies via methods used in [FrPe, LaWä].)

In [Lar2], another generalization of Wythoff Nim is studied, namely the family of Generalized Diagonal Wythoff Nim games and a so-called split of sequences of ordered pairs is defined. In particular a sequence of pairs $\left(\left(a_{i}, b_{i}\right)\right)$ is said to split if $(4)$ is not satisfied but (5) is (for some $l \geq 2$ ). In that paper one conjectures quite remarkable asymptotic 'splits' of $P$-positions for certain games - supported by numerous computer simulations and figures. However, the only proof of a 'splitting' of $P$-positions given in that paper is the much weaker statement that (4) is not satisfied, and it is only given for one particular game called $(1,2) \mathrm{GDWN}$ - a game which extends the diagonal options of Wythoff Nim and also allows moves of the types $(x, y) \rightarrow(x-i, y-2 i), x \geq i>0, y \geq$ $2 i>0$ and $(x, y) \rightarrow(x-2 i, y-i), x \geq 2 i>0, y \geq i>0$.

Remark 1. In this paper we have proved that the 'upper' (above the main diagonal) Ppositions of $\mathrm{W}^{2}$ split. I am not aware of any other such result, of a split of 'the upper' $P$-positions of an impartial game, in particular not on a variation of Wythoff Nim. (if we drop the 'upper' condition then one may obviously regard the P-positions of, for example, Wythoff Nim as a splitting sequence, see [Lar2]).

At the end of this section, we provide tables of the first few $P$-positions for $\mathrm{W}^{4}, \mathrm{~W}^{5}$ and $\mathrm{W}^{6}$ respectively. (It is interesting to note that the apparent simplicity of the set $\mathcal{R}_{3}$ does not seem to reappear for larger $k$.) As an appetizer for future research on Blocking- $k$ Wythoff Nim, let us motivate the conjectured asymptote of (4) in Table 1 for the case $\mathrm{W}^{4}$, that is that $\lim _{i \rightarrow \infty} \frac{b_{i}}{a_{i}}$ exists and equals $\sqrt{2}+1$. If (4) holds, with $\lim a_{i} / i=\alpha$ and $\lim b_{i} / i=\beta$ real numbers, then, by Proposition 1.2 , also $\alpha^{-1}+\beta^{-1}=4$ holds. Also, by Table 2, one hypothesis is that $\delta_{n}=b_{n}-a_{n}=n / 2+O(1)$, where $O(1)$ denotes some bounded function. This gives $\beta-\alpha=1 / 2$ and so, by elementary algebra we get

$$
\lim _{i \rightarrow \infty} \frac{b_{i}}{a_{i}}=\frac{\beta}{\alpha}=\sqrt{2}+1
$$

Acknowledgment. I wish to thank the anonymous referee for several useful remarks. 


\begin{tabular}{l||c|c|l|l|}
$k=$ & $l=1$ & $l=2$ & $l=3$ & $l=4$ \\
\hline 1 & $\phi$ & & & \\
\hline 2 & $\phi$ & 2 & & \\
\hline 3 & 2 & & & \\
\hline 4 & $1+\sqrt{2}$ & & & \\
\hline 5 & 1.476 & 2.5 & & \\
\hline 6 & 1.28 & 2.0 & 2.5 & \\
\hline 7 & 2.5 & & & \\
\hline 8 & 2.0 & 2.5 & & \\
\hline 9 & 1.34 & 2.5 & & \\
\hline 10 & 1.59 & 2.0 & 2.5 & \\
\hline 11 & 2.5 & & & \\
\hline 12 & 1.74 & 2.5 & & \\
\hline 13 & 1.2 & 2.5 & & \\
\hline 14 & 2.5 & & & \\
\hline 15 & $1+\sqrt{2}$ & 2.58 & & \\
\hline 16 & 1.426 & 2.5 & 2.6 & \\
\hline 17 & 1.12 & 2.0 & 2.5 & 2.6 \\
\hline 18 & 2.35 & 2.5 & 2.6 & \\
\hline 19 & 1.88 & 2.5 & 2.6 & \\
\hline 20 & 1.28 & 2.5 & 2.6 & \\
\hline
\end{tabular}

Table 1: The entries in this table are the estimated/conjectured quotients $\lim _{i \rightarrow \infty} \frac{b_{i}^{j}}{a_{t_{i}^{j}}}$ for $j \in\{1,2, \ldots, l\}$ and the respective game $\mathrm{W}^{k}$. The cases $k=2,3$ are resolved in Theorem 1.1 and $k=1$ is Wythoff Nim, $\phi=\frac{\sqrt{5}+1}{2}$. 

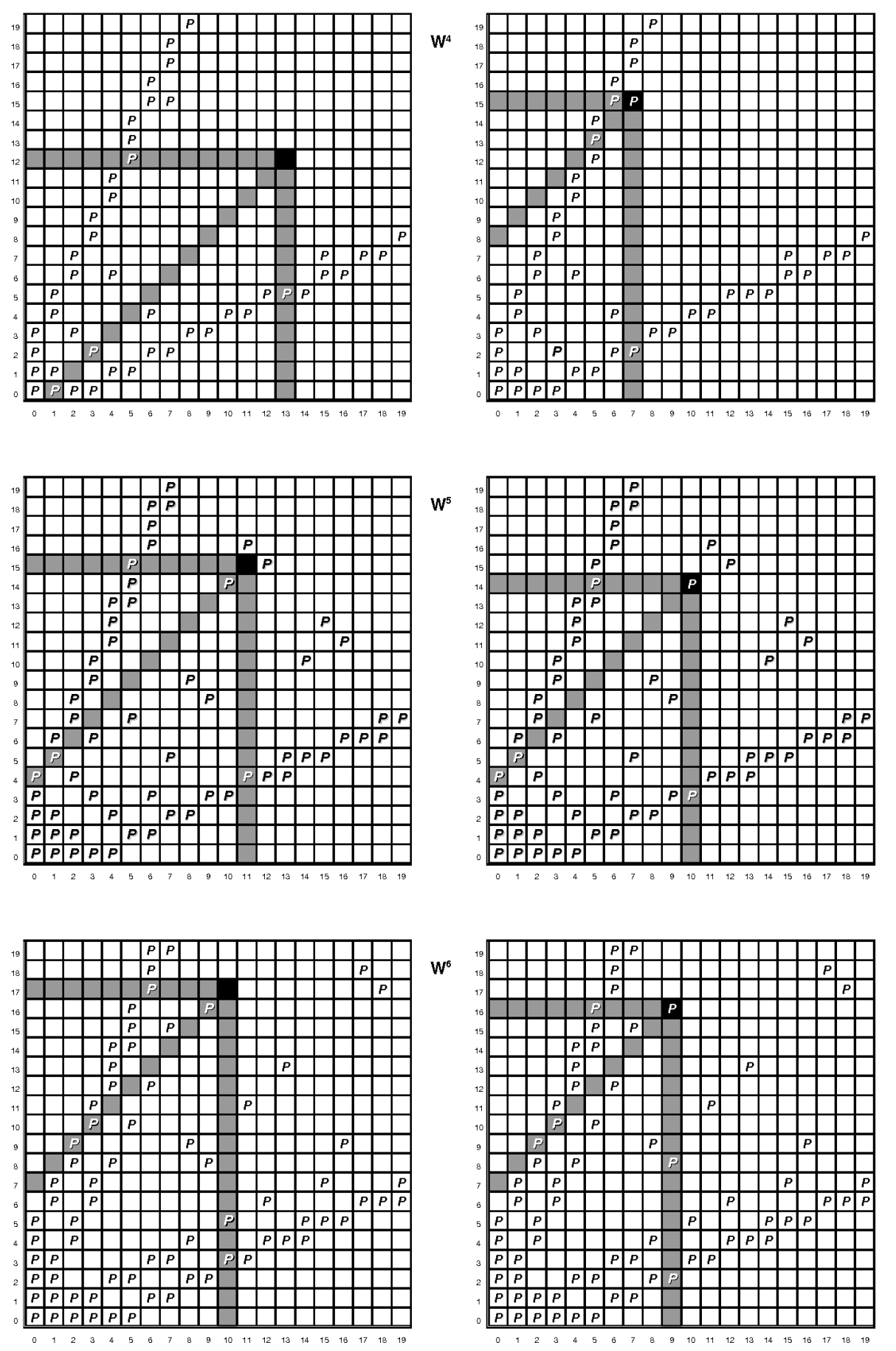

Figure 2: The figures represent the continuation of Figure 1 to the games $\mathrm{W}^{4}, \mathrm{~W}^{5}$ and $\mathrm{W}^{6}$ respectively (see also Table 2,3 and 4 ) . 


\begin{tabular}{l|c|c|c||c|c|c|c||c|c|c|c}
$n$ & $a_{n}$ & $b_{n}$ & $\delta_{n}$ & $n$ & $a_{n}$ & $b_{n}$ & $\delta_{n}$ & $n$ & $a_{n}$ & $b_{n}$ & $\delta_{n}$ \\
\hline 0 & 0 & 0 & 0 & 30 & 10 & 25 & 15 & 60 & 20 & 50 & 30 \\
1 & 0 & 1 & 1 & 31 & 10 & 26 & 16 & 61 & 21 & 51 & 30 \\
2 & 0 & 2 & 2 & 32 & 11 & 26 & 15 & 62 & 21 & 52 & 31 \\
3 & 0 & 3 & 3 & 33 & 11 & 27 & 16 & 63 & 22 & 53 & 31 \\
4 & 1 & 1 & 0 & 34 & 11 & 28 & 17 & 64 & 22 & 54 & 32 \\
5 & 1 & 4 & 3 & 35 & 12 & 29 & 17 & 65 & 22 & 55 & 33 \\
6 & 1 & 5 & 4 & 36 & 12 & 30 & 18 & 66 & 23 & 55 & 32 \\
7 & 2 & 3 & 1 & 37 & 12 & 31 & 19 & 67 & 23 & 56 & 33 \\
8 & 2 & 6 & 4 & 38 & 13 & 31 & 18 & 68 & 23 & 57 & 34 \\
9 & 2 & 7 & 5 & 39 & 13 & 32 & 19 & 69 & 24 & 58 & 34 \\
10 & 3 & 8 & 5 & 40 & 13 & 33 & 20 & 70 & 24 & 59 & 35 \\
11 & 3 & 9 & 6 & 41 & 14 & 34 & 20 & 71 & 24 & 60 & 36 \\
12 & 4 & 6 & 2 & 42 & 14 & 35 & 21 & 72 & 25 & 60 & 35 \\
13 & 4 & 10 & 6 & 43 & 14 & 36 & 22 & 73 & 25 & 61 & 36 \\
14 & 4 & 11 & 7 & 44 & 15 & 37 & 22 & 74 & 25 & 62 & 37 \\
15 & 5 & 12 & 7 & 45 & 15 & 38 & 23 & 75 & 26 & 63 & 37 \\
16 & 5 & 13 & 8 & 46 & 16 & 37 & 21 & 76 & 26 & 64 & 38 \\
17 & 5 & 14 & 9 & 47 & 16 & 39 & 23 & 77 & 27 & 65 & 38 \\
18 & 6 & 15 & 9 & 48 & 16 & 40 & 24 & 78 & 27 & 66 & 39 \\
19 & 6 & 16 & 10 & 49 & 17 & 41 & 24 & 79 & 27 & 67 & 40 \\
20 & 7 & 15 & 8 & 50 & 17 & 42 & 25 & 80 & 28 & 67 & 39 \\
21 & 7 & 17 & 10 & 51 & 17 & 43 & 26 & 81 & 28 & 68 & 40 \\
22 & 7 & 18 & 11 & 52 & 18 & 43 & 25 & 82 & 28 & 69 & 41 \\
23 & 8 & 19 & 11 & 53 & 18 & 44 & 26 & 83 & 29 & 70 & 41 \\
24 & 8 & 20 & 12 & 54 & 18 & 45 & 27 & 84 & 29 & 71 & 42 \\
25 & 8 & 21 & 13 & 55 & 19 & 46 & 27 & 85 & 29 & 72 & 43 \\
26 & 9 & 21 & 12 & 56 & 19 & 47 & 28 & 86 & 30 & 72 & 42 \\
27 & 9 & 22 & 13 & 57 & 19 & 48 & 29 & 87 & 30 & 73 & 43 \\
28 & 9 & 23 & 14 & 58 & 20 & 48 & 28 & 88 & 30 & 74 & 44 \\
29 & 10 & 24 & 14 & 59 & 20 & 49 & 29 & 89 & 31 & 75 & 44
\end{tabular}

Table 2: The first few $P$-positions of $\mathrm{W}^{4},\left\{a_{n}, b_{n}\right\}$, and the corresponding differences $\delta_{n}:=b_{n}-a_{n}$. 


\begin{tabular}{c|c|c|c||c|c|c|c||c|c|c|c}
$n$ & $a_{n}$ & $b_{n}$ & $\delta_{n}$ & $n$ & $a_{n}$ & $b_{n}$ & $\delta_{n}$ & $n$ & $a_{n}$ & $b_{n}$ & $\delta_{n}$ \\
\hline 0 & 0 & 0 & 0 & 30 & 8 & 21 & 13 & 60 & 17 & 24 & 7 \\
1 & 0 & 1 & 1 & 31 & 8 & 22 & 14 & 61 & 17 & 43 & 26 \\
2 & 0 & 2 & 2 & 32 & 8 & 23 & 15 & 62 & 17 & 44 & 27 \\
3 & 0 & 3 & 3 & 33 & 9 & 23 & 14 & 63 & 17 & 45 & 28 \\
4 & 0 & 4 & 4 & 34 & 9 & 24 & 15 & 64 & 18 & 46 & 28 \\
5 & 1 & 1 & 0 & 35 & 9 & 25 & 16 & 65 & 18 & 47 & 29 \\
6 & 1 & 2 & 1 & 36 & 10 & 14 & 4 & 66 & 18 & 48 & 30 \\
7 & 1 & 5 & 4 & 37 & 10 & 26 & 16 & 67 & 19 & 25 & 6 \\
8 & 1 & 6 & 5 & 38 & 10 & 27 & 17 & 68 & 19 & 48 & 29 \\
9 & 2 & 4 & 2 & 39 & 10 & 28 & 18 & 69 & 19 & 49 & 30 \\
10 & 2 & 7 & 5 & 40 & 11 & 16 & 5 & 70 & 19 & 50 & 31 \\
11 & 2 & 8 & 6 & 41 & 11 & 28 & 17 & 71 & 20 & 29 & 9 \\
12 & 3 & 3 & 0 & 42 & 11 & 29 & 18 & 72 & 20 & 51 & 31 \\
13 & 3 & 6 & 3 & 43 & 11 & 30 & 19 & 73 & 20 & 52 & 32 \\
14 & 3 & 9 & 6 & 44 & 12 & 15 & 3 & 74 & 20 & 53 & 33 \\
15 & 3 & 10 & 7 & 45 & 12 & 31 & 19 & 75 & 21 & 31 & 10 \\
16 & 4 & 11 & 7 & 46 & 12 & 32 & 20 & 76 & 21 & 53 & 32 \\
17 & 4 & 12 & 8 & 47 & 12 & 33 & 21 & 77 & 21 & 54 & 33 \\
18 & 4 & 13 & 9 & 48 & 13 & 33 & 20 & 78 & 21 & 55 & 34 \\
19 & 5 & 7 & 2 & 49 & 13 & 34 & 21 & 79 & 22 & 30 & 8 \\
20 & 5 & 13 & 8 & 50 & 13 & 35 & 22 & 80 & 22 & 56 & 34 \\
21 & 5 & 14 & 9 & 51 & 14 & 36 & 22 & 81 & 22 & 57 & 35 \\
22 & 5 & 15 & 10 & 52 & 14 & 37 & 23 & 82 & 22 & 58 & 36 \\
23 & 6 & 16 & 10 & 53 & 14 & 38 & 24 & 83 & 23 & 58 & 35 \\
24 & 6 & 17 & 11 & 54 & 15 & 38 & 23 & 84 & 23 & 59 & 36 \\
25 & 6 & 18 & 12 & 55 & 15 & 39 & 24 & 85 & 23 & 60 & 37 \\
26 & 7 & 18 & 11 & 56 & 15 & 40 & 25 & 86 & 24 & 61 & 37 \\
27 & 7 & 19 & 12 & 57 & 16 & 41 & 25 & 87 & 24 & 62 & 38 \\
28 & 7 & 20 & 13 & 58 & 16 & 42 & 26 & 88 & 24 & 63 & 39 \\
29 & 8 & 9 & 1 & 59 & 16 & 43 & 27 & 89 & 25 & 63 & 38
\end{tabular}

Table 3: The first few $P$-positions of $\mathrm{W}^{5},\left\{a_{n}, b_{n}\right\}$, and the corresponding differences $\delta_{n}:=b_{n}-a_{n}$. 


\begin{tabular}{l|c|c|c||c|c|c|c||c|c|c|c}
$n$ & $a_{n}$ & $b_{n}$ & $\delta_{n}$ & $n$ & $a_{n}$ & $b_{n}$ & $\delta_{n}$ & $n$ & $a_{n}$ & $b_{n}$ & $\delta_{n}$ \\
\hline 0 & 0 & 0 & 0 & 30 & 6 & 19 & 13 & 60 & 13 & 36 & 23 \\
1 & 0 & 1 & 1 & 31 & 7 & 15 & 8 & 61 & 14 & 27 & 13 \\
2 & 0 & 2 & 2 & 32 & 7 & 19 & 12 & 62 & 14 & 37 & 23 \\
3 & 0 & 3 & 3 & 33 & 7 & 20 & 13 & 63 & 14 & 38 & 24 \\
4 & 0 & 4 & 4 & 34 & 7 & 21 & 14 & 64 & 14 & 39 & 25 \\
5 & 0 & 5 & 5 & 35 & 8 & 9 & 1 & 65 & 15 & 30 & 15 \\
6 & 1 & 1 & 0 & 36 & 8 & 22 & 14 & 66 & 15 & 39 & 24 \\
7 & 1 & 2 & 1 & 37 & 8 & 23 & 15 & 67 & 15 & 40 & 25 \\
8 & 1 & 3 & 2 & 38 & 8 & 24 & 16 & 68 & 15 & 41 & 26 \\
9 & 1 & 6 & 5 & 39 & 9 & 16 & 7 & 69 & 16 & 32 & 16 \\
10 & 1 & 7 & 6 & 40 & 9 & 24 & 15 & 70 & 16 & 42 & 26 \\
11 & 2 & 4 & 2 & 41 & 9 & 25 & 16 & 71 & 16 & 43 & 27 \\
12 & 2 & 5 & 3 & 42 & 9 & 26 & 17 & 72 & 16 & 44 & 28 \\
13 & 2 & 8 & 6 & 43 & 10 & 20 & 10 & 73 & 17 & 18 & 1 \\
14 & 2 & 9 & 7 & 44 & 10 & 27 & 17 & 74 & 17 & 31 & 14 \\
15 & 3 & 6 & 3 & 45 & 10 & 28 & 18 & 75 & 17 & 44 & 27 \\
16 & 3 & 7 & 4 & 46 & 10 & 29 & 19 & 76 & 17 & 45 & 28 \\
17 & 3 & 10 & 7 & 47 & 11 & 11 & 0 & 77 & 17 & 46 & 29 \\
18 & 3 & 11 & 8 & 48 & 11 & 22 & 11 & 78 & 18 & 35 & 17 \\
19 & 4 & 8 & 4 & 49 & 11 & 29 & 18 & 79 & 18 & 47 & 29 \\
20 & 4 & 12 & 8 & 50 & 11 & 30 & 19 & 80 & 18 & 48 & 30 \\
21 & 4 & 13 & 9 & 51 & 11 & 31 & 20 & 81 & 18 & 49 & 31 \\
22 & 4 & 14 & 10 & 52 & 12 & 21 & 9 & 82 & 19 & 37 & 18 \\
23 & 5 & 10 & 5 & 53 & 12 & 32 & 20 & 83 & 19 & 49 & 30 \\
24 & 5 & 14 & 9 & 54 & 12 & 33 & 21 & 84 & 19 & 50 & 31 \\
25 & 5 & 15 & 10 & 55 & 12 & 34 & 22 & 85 & 19 & 51 & 32 \\
26 & 5 & 16 & 11 & 56 & 13 & 13 & 0 & 86 & 20 & 40 & 20 \\
27 & 6 & 12 & 6 & 57 & 13 & 25 & 12 & 87 & 20 & 52 & 32 \\
28 & 6 & 17 & 11 & 58 & 13 & 34 & 21 & 88 & 20 & 53 & 33 \\
29 & 6 & 18 & 12 & 59 & 13 & 35 & 22 & 89 & 20 & 54 & 34
\end{tabular}

Table 4: The first few $P$-positions of $\mathrm{W}^{6},\left\{a_{n}, b_{n}\right\}$, and the corresponding differences $\delta_{n}:=b_{n}-a_{n}$.

\section{References}

[ANW07] M. H. Albert, R. J. Nowakowski, D. Wolfe Lessons in Play: In Introduction to Combinatorial Game Theory. A K Peters Ltd.(2007).

[BCG82] E. R. Berlekamp, J. H. Conway, R.K. Guy, Winning ways, 1-2 Academic Press, London (1982). Second edition, 1-4. A. K. Peters, Wellesley/MA $(2001 / 03 / 03 / 04)$. 
[Bea26] S. Beatty, Problem 3173, Amer. Math. Monthly, 33 (1926) 159.

[Con76] J.H.Conway, On Numbers and Games, Academic Press, London (1976). Second edition, A.K.Peters, Wellesley/MA (2001).

[Bou02] C.L. Bouton, Nim, a game with a complete mathematical theory, The Annals of Math. Princeton (2) 3 (1902), 35-39.

[Fra73] A.S. Fraenkel, Complementing and exactly covering sequences, J. Combinatorial Theory Ser. A 14 (1973), 8-20.

[Fra82] A. S. Fraenkel, How to beat your Wythoff games' opponent on three fronts, Amer. Math. Monthly89 (1982) 353-361.

[Fra04] A. S. Fraenkel, Complexity, appeal and challenges of combinatorial games. Theoret. Comp. Sci., 313 (2004) 393-415.

[FrPe] A.S. Fraenkel, U. Peled, Harnessing the Unwieldy MEX Function, preprint, http://www.wisdom.weizmann.ac.il/ fraenkel/Papers/ Harnessing.The.Unwieldy.MEX.Function_2.pdf.

[Gur10] V. Gurvich, Further generalizations of Wythoff's game and minimum excludant function, RUTCOR Research Report, 16-2010, Rutgers University.

[GaSt04] H. Gavel and P. Strimling, Nim with a Modular Muller Twist, Integers: Electr. Jour. Comb. Numb. Theo. 4 (2004).

[Gra73] R.L. Graham, Covering the positive integers by disjoint sets of the form $\lfloor n \alpha+\beta\rfloor: n=1,2, \ldots$, J. Combinatorial Theory Ser. A 15 (1973), 354-358.

[Heg1] On $m$-covering families of Beatty sequences with irrational moduli, preprint.

[HeLa06] P. Hegarty and U. Larsson, Permutations of the natural numbers with prescribed difference multisets, Integers 6 (2006), Paper A3, 25pp.

[HoRe01] Holshouser, A. and H. Reiter, Blocking Nim, November 2001, problem 714, College Mathematics Journal, Vol 32, No 5.

[HoRe] A. Holshouser and H. Reiter, Three Pile Nim with Move Blocking, http://citeseer.ist.psu.edu/470020.html.

[HoRe1] A. Holshouser and H. Reiter, Blocking combinatorial games, http://math.uncc.edu/ hbreiter/BlockComb2.pdf

[Lar09] U. Larsson, 2-pile Nim with a Restricted Number of Move-size Imitations, Integers 9 (2009), Paper G4, pp 671-690.

[Lar1] U. Larsson, Restrictions of $m$-Wythoff Nim and $p$-complementary Beatty sequences, to appear in Games of no Chance 4.

[LaWä] U. Larsson, J. Wästlund, Maharajah Nim, Wythoff's Queen meets the Knight, preprint.

[Lar2] Urban Larsson, A Generalized Diagonal Wythoff Nim, to appear in Integers.

[SmSt02] F. Smith and P. Stănică, Comply/Constrain Games or Games with a Muller Twist, Integers, 2, (2002).

[Wyt07] W.A.Wythoff. A modification of the game of Nim. Nieuw Arch. Wisk. 7 (1907) 199-202. 